\title{
Induction Therapy, HLA Mismatching, and Standard-Risk Kidney Transplantation
}

\author{
Mahmoud Nassar ${ }^{\mathrm{a}}$ Mohammed Elshafey ${ }^{\mathrm{b}}$ Carlos Gonzalez $^{\mathrm{a}} \quad$ Nso Nso $^{\mathrm{a}}$ \\ Mamdouh Mahdic Ingi Elsayed ${ }^{\mathrm{d}}$ \\ aMedicine Department, Icahn School of Medicine at Mount Sinai/NYC Health + Hospitals Queens, \\ New York, NY, USA; b Department of Obstetrics and Gynecology, University of Tennessee Health Science Center, \\ Memphis, TN, USA; 'Professor of Nephrology, Dean of Faculty of Medicine, Helwan University, Cairo, Egypt; \\ ${ }^{d}$ Renal Department, University Hospitals of North Midlands, Stoke-on-Trent, UK
}

\section{Dear Editor,}

We have read with great interest the research work titled "Outcomes of Interleukin-2 Receptor Antagonist Induction Therapy in Standard-Risk Renal Transplant Recipients Maintained on Tacrolimus: A Systematic Review and Meta-Analysis" conducted by Hatem Ali et al. [1].

The authors found no additive benefit for interleukin-2 induction therapy in the standard risk population. Similar results were shown in the previous registry and in retrospective studies $[2,3]$. The authors used a broad definition for standard-risk renal transplant which included HLA mismatches of $<5$.

Most of the studies included in the meta-analysis used a low-resolution 6-digit HLA mismatching technique. The current technology for HLA mismatching uses me- dium and high-resolution HLA mismatching to detect mismatching at the epitope and eplet levels. There is growing evidence that mismatching at the epitope and eplet levels might affect the outcomes [4]. This should be taken into consideration while assessing the immunological risk and decision about induction therapy.

Furthermore, there is emerging evidence that HLADQ mismatch has a pivotal role in kidney transplantation outcomes, and it should also be considered for assessment of the transplant immunological risk [5].

I agree with the authors that interleukin-2 induction therapy might not be needed in low-risk renal transplant patients. However, this decision should be taken after considering HLA-DQ mismatching and epitope and eplet levels via HLA mismatching.

\section{References}

1 Ali H, Mohamed MM, Sharma A, Fulop T, Halawa A. Outcomes of interleukin-2 receptor antagonist induction therapy in standardrisk renal transplant recipients maintained on tacrolimus: a systematic review and metaanalysis. Am J Nephrol. 2021;52(4):279-91.

2 Ali H, Sharma A, Halawa A. Effect of interleukin-2 receptor antibody induction therapy on survival in renal transplant patients receiving tacrolimus. Am J Nephrol. 2020;51(5):366-72.

3 Ali H, Mohiuddin A, Sharma A, Shaheen I, Kim JJ, El Kosi M, et al. Implication of interleukin-2 receptor antibody induction therapy in standard risk renal transplant in the tacrolimus era: a meta-analysis. Clin Kidney J. 2019;12(4):592-9.
4 Buttigieg J, Ali H, Sharma A, Halawa A. Positive luminex and negative flow cytometry in kidney transplantation: a systematic review and meta-analysis. Nephrol Dial Transplant. 2019;34(11):1950-60.

5 Tambur AR. HLA-DQ antibodies: are they real? Are they relevant? Why so many? Curr Opin Organ Transplant. 2016;21(4):441-6.
Correspondence to:

Mahmoud Nassar, dr.nassar@aucegypt.edu Ingi Elsayed, ingi.elsayed@uhnm.nhs.uk 